\title{
Electrolyte-Moderated Interactions in Water/Oil Microemulsions
}

\author{
BRIAN BEDWELL AND ERDOGAN GULARI' \\ Department of Chemical Engineering, University of Michigan, Ann Arbor, Michigan, 48109
}

Received January 31, 1984; accepted Apri1 25, 1984

\begin{abstract}
Interactions between the disperse phase droplets of water-in-oil (W/O) microemulsions of AerosolOT are profoundly affected by the addition of small amounts of electrolyte. We find that the effect of added electrolyte in W/O microemulsions is opposite to that which is observed in aqueous micellar solutions and $\mathrm{O} / \mathrm{W}$ microemulsions, here the addition of electrolyte decreases attractive interactions rather than increasing them. Dynamic light scattering measurements show that the addition of salt decreases (and even eliminates) the dependence of the diffusion coefficient on disperse phase concentration. Time-averaged intensity measurements and shear viscosity measurements provide further evidence of increased hard-sphere-like behavior as the salt concentration increases. Analysis of the time-averaged intensity using a formalism developed by A. A. Calje, W. G. M. Agterof, and A. Vrij ("Micellizations, Solubilization, and Microemulsions" (K. L. Mittal, Ed.), Vol. 2, Plenum, New York, 1977) suggests that the attractive part of the interaction potential is significantly larger than that expected between pure water droplets. The attractive potential decreases as the electrolyte concentration increases. Based on interactions between fluctuating dipoles, a semiquantitative explanation for the effect of salt on attractive interactions is provided. () 1984 Academic Press, Inc.
\end{abstract}

\section{INTRODUCTION}

Water-in-oil (W/O) microemulsions of AOT (bis(2-ethylhexyl)-sulfosuccinate sodium salt) in nonpolar solvents are profoundly affected by the addition of small amounts of electrolyte. Kuneida and Shinoda (1) have shown that the domain of existence (in the ternary phase diagram) of these microemulsions is significantly reduced when there are inorganic salts present in the system. A number of investigators have addressed the question of the effect of extra components on the phase behavior of microemulsions of AOT in nonpolar solvents (1-8). We are particularly interested in the effect of electrolyte. Inorganic salts added to these systems decrease the solubility of water and shrink the $\mathrm{L}$ region of the ternary phase diagram (1-4).

AOT forms globular micelles in nonpolar solvents and spherical droplets in W/O microemulsions (9-11). Eicke and Shepherd

\footnotetext{
${ }^{1}$ Author to whom correspondence should be addressed.
}

(12) measured the dielectric increment due to micelles and $\mathrm{W} / \mathrm{O}$ microemulsions as a function of water content and electrolyte added to the water. They found that water increased the magnitude of the dipole generated in an aggregate by an external electric field. Electrolyte added to the system decreased the magnitude of the dipole moment generated in a W/O microemulsion macroparticle. Eicke et al. (13) demonstrated that water and electrolyte is exchanged between suspended microemulsion droplets, by mixing two microemulsions containing two different aqueous solutions which became fluorescent when combined. A similar conclusion was reached by Fletcher and Robinson (14). Eicke and Markovic (15) measured the characteristic times for electrically induced birefringence. Two decay times were apparent. They postulated two mechanisms for the relaxation of a dipole in a macroparticle: Brownian reorientation and diffusion of ions in the aqueous core. Wong et al. (16) used proton and sodium-23 NMR to investigate $W / O$ 
microemulsions of AOT in heptane. Their results show that in small micelles the water is immobilized, but becomes more mobile as the amount of water in each droplet increases. They found that at low water content the sodium ions were bound to the anionic head groups. At higher water content (water-toAOT molar ratio $\left.\left(N_{\mathrm{w}} / N_{\mathrm{aot}}\right)=49\right)$, they estimate that about $28 \%$ of the sodium ions in the microemulsion particle were dissociated. The characteristic structures in these solutions appear to be spherical aqueous electrolyte droplets, whose cores can communicate.

A number of investigators have addressed the problem of interacting droplets using quaternary $\mathrm{W} / \mathrm{O}$ microemulsions as an experimental system. For microemulsions of cyclohexane, sodium dodecyl sulfate, pentanol, and water, neutron scattering (17), ultracentrifugation (18), dynamic light scattering (DLS) (18), and average intensity light scattering (19) experiments all indicate that the droplets behave like hard spheres. DLS measurements on similar systems made with butanol instead of pentanol revealed a different pattern. The apparent diffusion coefficient decreased by a factor of 8 over the volume fraction range 0 to 0.1 , whereas the pentanol system showed essentially no change.

Lemaire et al. (20) explained this dependence of the interaction between droplets on the alcohol chain length as being due to the interpenetration of the surface layers of the microemulsion droplets, short chains allowing deeper penetration than long chains. Brunetti et al. (21) confirmed that at low volume fraction this theory was successful. Cazabat et al. (22) found that $\mathrm{NaCl}$ added to the water core of $\mathrm{W} / \mathrm{O}$ microemulsions of a toluene, SDS, and butanol system reduced the apparent volume fraction dependence of the diffusion coefficient measured by DLS. The pattern that these authors observed with $\mathrm{NaCl}$ is very similar to that which we obtain below.

Calje et al. (23) analyzed the results of average intensity light scattering experiments from concentrated systems using Carnahan and Starling's (24) results for a hard sphere fluid. By adding a van der Waals-type attractive perturbation to the hard-sphere formulation they related the osmotic compressibility (and thus the intensity of scattered light) to the individual droplet parameters by

$$
\begin{array}{r}
\frac{d}{d c}\left(\frac{\pi}{R_{\mathrm{B}} T}\right)=\frac{(1+2 w c)^{2}-w^{3} c^{3}(4-w c)}{M(1-w c)^{4}} \\
-\frac{2 A N_{\mathrm{av}} c}{R_{\mathrm{B}} T M^{2}}
\end{array}
$$

where $A=$ van der Waals constant,

$$
\begin{aligned}
w & =\frac{4}{3} \pi\left(R_{\mathrm{hs}}\right)^{3} \frac{N_{\mathrm{av}}}{M}, \\
M & =\text { molecular weight, } \\
R_{\mathrm{hs}} & =\text { hard-sphere radius }
\end{aligned}
$$

$N_{\text {av }}$ is Avogadro's number, $R_{\mathrm{B}}$ is the gas constant, and $T$ is the absolute temperature. With this formalism, intensity measurements made as a function of disperse phase concentration may be used to estimate $R_{\mathrm{hs}}, M$, and $A$. This theory assumes that the solute particle's most significant features are the hard-sphere repulsion and a van der Waals attraction. Testing this procedure with a W/O microemulsion, these authors concluded that the value of $A$ was unrealistically large for water droplets. If these droplets interact through mechanisms other than a simple van der Waals force, this may not be the case.

Scattering experiments are attractive methods for investigating the properties of colloidal solutions. Small-angle neutron scattering, small-angle X-ray scattering, and average intensity light scattering can all reveal information about the characteristic distances in condensed phases, while DLS measures characteristic times. In general, these microscopic properties can arise from different types of physical situations. In the case of microemulsions, the colloidal particles of interest are aggregates of smaller molecules. The integrity of these aggregates is not assured; they can increase or decrease in size through the gain or loss of small molecules. In the event that such aggregates exist in solution, they can 
interact with each other resulting in a correlation between neighboring particle positions. The interpretation of scattering data becomes difficult in the context of these combined effects. A perceived characteristic distance from an experiment could be due to an individual particle size or to a typical interparticle spacing. A measured correlation time in the DLS experiment could be due to the independent Brownian motion of a microemulsion droplet and/or to the cooperative motion of interacting droplets.

A further, compounding problem is polydispersity. Currently there are two common methods for dealing with polydispersity arising in the DLS experiment: the cumulants method (25) and the direct methods $(26,27)$, such as the histogram method (27).

As a part of our continuing research to understand microemulsion structure and interactions, we undertook a detailed study of structure and interparticle interactions in concentrated $\mathrm{W} / \mathrm{O}$ microemulsion systems with and without extra electrolyte. Water/ AOT/heptane was chosen in order to eliminate the complicating effect of the cosurfactant needed by the single tailed surfactants. In this paper, we report the results of our light scattering and viscosity studies, and propose a semiquantitative explanation for the origin of the attractive force between the disperse phase droplets in the $\mathrm{W} / \mathrm{O}$ microemulsion.

\section{EXPERIMENTAL}

\section{Materials}

Heptane and $\mathrm{NaCl}$ were reagent grade. Water was doubly distilled, deionized. AOT was Fluka ( $98 \%$ pure), which was further purified in order to remove trace amounts of inorganic impurity and adsorbed water. The dried and purified AOT was stored over phosphorous pentoxide in a dessicator.

\section{Microemulsion Preparation}

Water-in-oil microemulsions were prepared by adding water or $\mathrm{NaCl}$ brine to a concentrated solution of AOT in heptane. When the additive was pure water, the system would become one phase in a short time $(<1 \mathrm{~min})$ with gentle shaking. Brine microemulsions took longer to become one phase. The time required to reach this one-phase equilibrium condition increased with electrolyte concentration in the brine and decreased with increasing AOT concentration in heptane. Ultrasound and moderate heating did not have a significant effect on the rate of equilibration for the heavily salted systems. These concentrated solutions were then diluted with heptane (water saturated) to generate a series of less-concentrated microemulsions.

The systems containing more-concentrated $\mathrm{NaCl}$ brine required as much as 2 weeks to become one phase. Once this one-phase condition was reached, the systems were stable for months.

Droplet volume fractions for these solutions were estimated from measured solution densities assuming that the solute particles contained all of the water and AOT and that the heptane continuum was unaltered by the presence of the solute.

All light scattering and viscometry experiments were performed on stable one-phase systems at $25^{\circ} \mathrm{C}$.

\section{Light Scattering}

Both dynamic light scattering (DLS) experiments and average intensity measurements were made using apparatus we have described previously (28).

Light scattering samples were filtered through $0.2-\mu \mathrm{m}$ Millipore filters and centrifuged in order to eliminate suspended dust. Data collection in the light scattering experiment was controlled by a minicomputer, so that the effect of residual dust could be minimized. Samples were taken for short times (1-3 sec) and the scattered intensity was compared to the average. If the measured intensity was different from the average by several percents, then that sample was discarded since dust in the sample volume significantly alters the scattered signal. Sam- 
ples which passed this test, were accumulated in the controlling computer's memory.

Average intensity light scattering measurements were made at an angle of 90 degrees. DLS measurements were made at angles ranging from 16 to 90 degrees.

Osmotic compressibilities were calculated from the light scattering intensities measured at 90 degrees scattering angle using

$$
R_{90}=2 \pi^{2} n^{2}\left(\frac{\partial n}{\partial c}\right)^{2} \lambda^{-4} k T c\left(\frac{\partial \pi}{\partial c}\right)^{-1}
$$

Here $R$ is the excess scattered intensity, $\lambda$ is the wavelength under vacuum, $n$ is the refractive index of the microemulsion, $k$ is the Boltzman constant, $c$ is the concentration $(\mathrm{g} /$ $\mathrm{ml}$ ) of the microemulsion droplets, and $\pi$ is the osmotic pressure. The diffusion coefficients were determined from nonlinear leastsquare analysis of the homodyne autocorrelation functions.

\section{Viscometry}

Solution kinematic viscosities, $\mu$, were measured with appropriate Canon-Fenske viscometers of size 25,50 , or 100 according to the viscosity of the sample. The solution densities, $\rho$, were measured with pycnometers. The shear viscosity, $\eta$, was then calculated from the relation $\eta=\mu \rho$. The Canon-Fenske viscometer does not allow for control of the shear rate, so the assumption that the microemulsions were Newtonian had to be made in order to interpret the measurements as shear viscosities. In fact, the microemulsions without any $\mathrm{NaCl}$ appeared to be somewhat non-Newtonian, while the addition of $\mathrm{NaCl}$ appeared to bring about more Newtonian behavior.

\section{RESULTS AND DISCUSSION}

Figure 1 shows the average diffusion coefficient from cumulants analysis of dynamic light scattering experiments on the three dilution series with constant water-to-AOT ratio of 8.4 and varying salt concentration. This data shows clearly that a small amount of

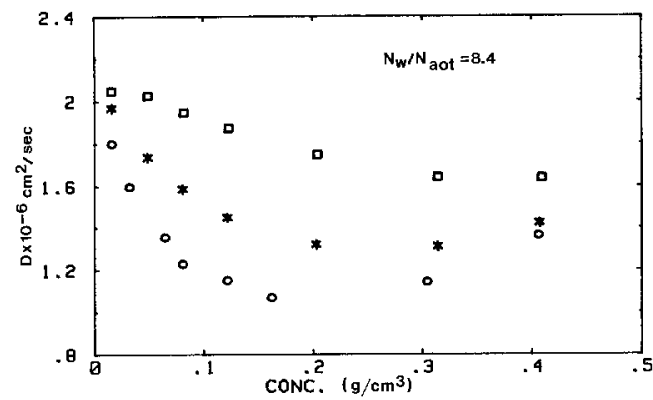

FIG. 1. Diffusion coefficient (from first cumulant) vs concentration for $N_{\mathrm{w}} / N_{\mathrm{aot}}=8.4$, no salt (O), 1\% salt (*), and $3 \%$ salt $(\square)$. The horizontal axis represents total solute concentration (AOT, water, and $\mathrm{NaCl}$ ). These measurements were made at a scattering angle of 16 degrees.

$\mathrm{NaCl}$ significantly alters the diffusion coefficient of the microemulsion particle in heptane. The negative initial slope of the purewater system is consistent with the behavior of macroparticles that experience attractions (29). The reduction of this curvature through the addition of $\mathrm{NaCl}$, exhibited by the 1 and $3 \%$ brine systems, suggests that such attractions are diminished by the $\mathrm{NaCl}$.

All three of these curves appear to have essentially the same intercept where the solute concentration becomes zero. At high dilution, the Stokes-Einstein relation becomes valid. Therefore, the common intercept suggests that all three systems contain particles of essentially the same size.

At higher concentrations, the theoretical basis for interpretation of dynamic light scattering data has not been developed sufficiently to distinguish unambiguously the relative contributions of the size and interaction effects. However, we can say that the lower diffusion coefficient in the unsalted system is consistent with an attractive force. Either the cooperative motion of neighboring particles slows down the characteristic Brownian motion and/or the formation of aggregates through an attraction results in a larger average particle size and hence a lower diffusion coefficient.

Viscosity measurements on these same systems support the assertion that the unsalted 
microemulsion experiences an attraction. The data in Fig. 2 shows the concentration dependence of the relative viscosity for dilution series with water-to-AOT ratio of 8.4 and salt concentrations of 0 and $3 \%$ by weight in water. The curves drawn near the data points are due to equations

$$
\begin{aligned}
& \eta_{\text {rel }}=\exp [2.5 \theta /(1-1.65 \theta)] \\
& \eta_{\text {rel }}=(1-1.35 \theta)^{-2.5} \\
& \eta_{\text {rel }}=\exp \left[\left(2.5 \theta+\mathrm{A}_{\mathrm{v}} \theta^{2}\right) /(1-1.65 \theta)\right],
\end{aligned}
$$

where $\eta_{\mathrm{rel}}=$ relative viscosity, $\theta=$ volume fraction of dispersed phase. The first two expressions represent two different theories for the hard sphere, volume fraction dependence of the relative viscosity. The third expression has an extra term to account for attractive interactions (30-34). The data for $3 \%$ brine system fall near the theoretical prediction for the viscosity of a monodisperse solution of hard spheres, while the data for unsalted system deviate significantly from this behavior. The observed increase in viscosity for the unsalted system is consistent with attractions between neighboring particles. The addition of an attractive term to Eq. [3] yields Eq. [5]. With this adjustment, the curve generated by Eq. [5] (with $A_{\mathrm{v}}$ $=20$ ) passes near the data for the unsalted solutions. We should point out that $A_{\mathrm{v}}$ is essentially an adjustable constant and as such the increased agreement is not surprising.

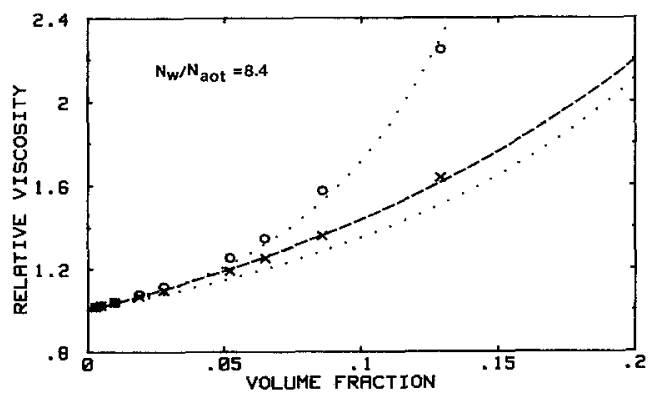

FIG. 2. Relative shear viscosity vs disperse phase volume fraction (AOT, water, and $\mathrm{NaCl}$ ) for fixed waterto-AOT ratio of 8.4. No salt $(O)$ and $3 \%$ salt $(X)$. The dotted lines are due to Eqs. [3] and [5]. The dashed line is due to Eq. [4].
A simple increase in the average particle size would not account for the increase in viscosity. The hard-sphere theory is independent of particle size. Increases in polydispersity would decrease the measured viscosity (35).

Deviation from a spherical shape is a plausible explanation for the increase in viscosity; but evidence from X-ray scattering compared to light scattering suggests that these particles are spherical at low concentration (28). Aggregates of spherical particles would result in an apparent shape change, so this shape-related explanation is consistent with the presence of an attractive force.

As indicated above, Calje et al. (23) have developed a procedure for estimating the hard-sphere radius, the molecular weight, and the van der Waals force constant from average intensity light scattering data (assuming that the colloidal particles are well characterized by a hard-sphere potential, perturbed by a long range attraction). Figure 3 shows the osmotic compressibility as a function of concentration for the systems with water to AOT ratio of 8.4 and salt concentrations of $0,1,3 \%$, respectively, along with the curves generated by fitting Eq. [1] to these data. The parameters resulting from these fits are given in Table I.

These results are again consistent with the notion that the pure water microemulsion experiences attractions which are moderated by the addition of $\mathrm{NaCl}$. The van der Waals constant, $A$, is reduced by $\mathrm{NaCl}$. We also note that the difference between the hardsphere radius and the hydrodynamic radius varies from a low of 4 to $7.5 \AA$. These differences are less than the fully extended length of the AOT molecule $\left(l_{\mathrm{c}}=11 \AA\right)$ but are reasonable if one assumes that the hardsphere radius extends all the way to the ester groups of the surfactants.

The DLS experiments and average intensity measurements of the osmotic compressibility were also performed on systems with waterto-AOT ratios of 4.2 and 16.8 with and without $1 \%$ salt in water. The behavior was 


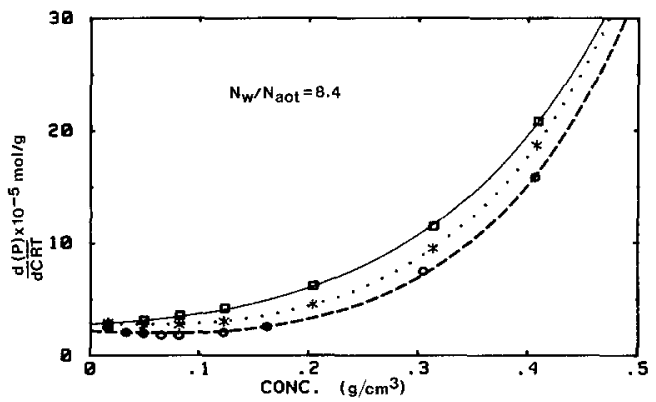

FIG. 3. Osmotic compressibility vs total solute concentration for fixed water-to-AOT ratio of 8.4 and salt concentrations of $0 \%(O), 1 \%(*)$, and $3 \%(\square)$. The lines are fits of Eq. [1].

very similar to that shown in Figs. 1 and 3. With both these systems the curvature is reduced upon the addition of $\mathrm{NaCl}$. The effect is not as pronounced as observed with the system containing 3\% brine. One feature of the osmotic compressibility data that should be noted is that there is curvature in the data near zero concentration, which is moderated by the addition of $\mathrm{NaCl}$. For unsalted systems the osmotic compressibility increases at low concentration, while the corresponding salted systems tend to approach the intercept with a slope closer to zero. For noninteracting solutes, the osmotic pressure behaves as a colligative property and as such is linearly dependent on concentration at high dilution. For particles which experience a long range attraction, this slope or virial coefficient is expected to be negative.

The intercept that we observe with this curvature in unsalted systems of 4.2 and 16.8 water-to-AOT ratio is inconsistent with the DLS results. For the DLS experiments we observed that the salted and unsalted systems had a common intercept, suggesting a hydrodynamic size at high dilution which was independent of salt content. For a similar

TABLE I

Results of Least-Square Fit of Osmotic Compressibility Data to Eq. [1]

\begin{tabular}{|c|c|c|c|c|c|}
\hline Sample & $\begin{array}{l}\text { No. points } \\
\text { used for } \\
\text { fitting }\end{array}$ & $\begin{array}{l}\text { Molecular } \\
\text { weight }\end{array}$ & $\begin{array}{c}\text { van der Waal's } \\
\text { constant } \\
\left(A \times 10^{9} \mathrm{~cm}^{3} \mathrm{erg}\right)\end{array}$ & $\begin{array}{l}\text { Hard-sphere } \\
\text { radius, } R_{\mathrm{has}} \\
(\AA)\end{array}$ & 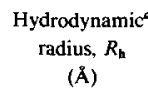 \\
\hline \multicolumn{6}{|l|}{$N_{\mathrm{w}} / N_{\mathrm{aot}}=8.4$} \\
\hline \multirow[t]{2}{*}{$0 \mathrm{wt} \% \mathrm{NaCl}$} & 9 & 45,669 & 8.2 & 24.0 & \\
\hline & 6 & 62,417 & 8.8 & 27.0 & \\
\hline \multirow[t]{2}{*}{$1 \mathrm{wt} \% \mathrm{NaCl}$} & 7 & 34,643 & 5.4 & 21.5 & \\
\hline & 6 & 36,362 & 5.5 & 22.0 & \\
\hline \multirow[t]{2}{*}{$3 \mathrm{wt} \% \mathrm{NaCl}$} & 7 & 35,610 & 2.9 & 21.5 & \multirow[t]{2}{*}{29.0} \\
\hline & 6 & 33,882 & 3.1 & 21.0 & \\
\hline \multicolumn{6}{|l|}{$N_{\mathrm{w}} / N_{\mathrm{aot}}=4.2$} \\
\hline \multirow[t]{2}{*}{0 wt $\% \mathrm{NaCl}$} & 9 & 12,915 & 2.6 & 15.0 & \\
\hline & 7 & 20,968 & 3.2 & 18.0 & \\
\hline \multirow[t]{2}{*}{$1 \mathrm{wt} \% \mathrm{NaCl}$} & 9 & 16,936 & 2.6 & 16.5 & \multirow[t]{2}{*}{21.3} \\
\hline & 7 & 18,335 & 2.7 & 17.0 & \\
\hline \multicolumn{6}{|l|}{$N_{\mathrm{w}} / N_{\mathrm{aot}}=16.8$} \\
\hline \multirow[t]{2}{*}{$0 \mathrm{wt} \% \mathrm{NaCl}$} & 9 & 107,320 & 16.0 & 32.0 & \\
\hline & 8 & 151,600 & 15.0 & 35.5 & \\
\hline \multirow[t]{2}{*}{$1 \mathrm{wt} \% \mathrm{NaCl}$} & 9 & 142,450 & 5.3 & 35.0 & \multirow[t]{2}{*}{39.0} \\
\hline & 8 & 149,870 & 3.7 & 36.0 & \\
\hline
\end{tabular}

\footnotetext{
${ }^{a}$ Hydrodynamic radius was calculated from the diffusion coefficient using the Stokes-Einstein relationship $D=\left(k T / 6 \pi R_{\mathrm{h}} \eta\right)$, where $\eta$ is the solvent viscosity and $k$ is the Boltzmann constant and $T$ is the absolute temperature.
} 
result in the average intensity experiment we would again expect a common intercept. This would be equivalent to having a molecular weight for the droplets which was independent of salt content. The larger value for the intercept corresponds to a lower molecular weight. This suggests that the unsalted system has less droplet integrity or that the continuum contains more water and AOT than in the salted system. Cabos and Delord (36) found that salt increased the characteristic droplet size at low volume fraction using neutron scattering. This is consistent with the results of our experiment, at low volume fraction. At higher volume fraction, we find the reverse. Electrolyte in the droplet increases the likelihood that water will remain in the droplet, in order to shield charged solute. This curvature at high dilution could be due to droplet break-up and/or interparticle attractions. In Table I, we report the results of fitting Eq. [1] to the intensity data for these systems with and without the points in the high dilution region. Note that without the high dilution points, the salted and unsalted systems have similar intercepts, indicating similar sizes. Lalanne et al. (37) have performed similar experiments for AOT W/O microemulsions in $\mathrm{CCl}_{4}$. There is evidence of this curvature in their data.

Systems with a water-to-AOT ratio of 4.2 , 8.4 , and 16.8 were all relatively monodisperse, with variances estimated by cumulants analysis of DLS measurements ranging from 0 to 0.06 . In order to elucidate the effect of $\mathrm{NaCl}$ on polydispersity, two new systems with water-to-AOT ratios of 4.2 and 12 were prepared. The salt concentrations ranged from 0 to $11 \%$ for the first system and 0 to $3 \%$ for the second system. For the brine solutions used in this section, the $\mathrm{NaCl}$ concentration was near the solubility limit for one-phase W/O microemulsions.

For the second system 5 microemulsions were prepared all with $N_{\mathrm{w}} / N_{\text {aot }}=12$. Pure water, $2,3,4$, and $5 \%$ brine were added to $50 \%$ AOT in heptane. The 5\% brine system did not become one phase after 2 weeks of gentle shaking. The others became one phase after shaking for various times, the water system almost immediately and the $4 \%$ after about a day. Upon dilution with heptane, the $4 \%$ brine system broke into two phases. The water, 2 and 3\% systems remained one phase with dilution. Figure 4 shows the average diffusion coefficient obtained by $\mathrm{cu}-$ mulant analysis of DLS measurements on dilutions of systems with no salt and $3 \%$ salt.

For the first system, several microemulsions were prepared from $50 \%$ AOT in heptane, with $N_{\mathrm{w}} / N_{\text {aot }}=4.2$. The $\mathrm{NaCl}$ content in the brine ranged from 0 to $11 \%$. All of these systems became one phase with shaking and remained one phase with dilution. The salted systems required more time to reach equilibrium. Average diffusion coefficients for some of these dilutions are presented in Fig. 5.

Figures 4 and 5 show the same trend observed in Fig. 1. The salted microemulsions have reduced concentration dependence in the average diffusion coefficient and for the highest salt concentration $(11 \%)$ system shown in Fig. 5 the slope of the diffusion coefficient with concentration is positive indicating that the repulsive interactions dominate. Table II shows the numerical results from the cumulants analysis for these systems. There are two trends in polydispersity revealed in these results: (i) $\mathrm{NaCl}$ reduces poly-

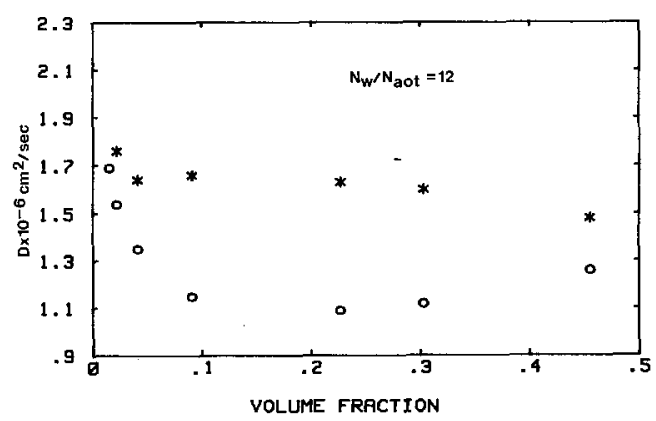

FIG. 4. Diffusion coefficient vs total solute concentration for water-to-AOT ratio of $12,0 \% \mathrm{NaCl}(O)$, and $3 \%$ $\mathrm{NaCl}(*)$. The scattering angle for these measurements was 90 degrees. 


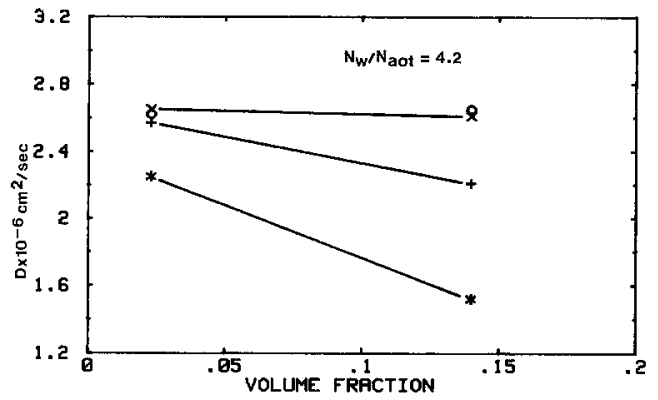

FIG. 5. Diffusion coefficient vs total solute concentration for fixed water-to-AOT ratio of 4.2 and salt concentrations of $0(*), 3(+), 7(\times)$, and $11 \%(O)$.

dispersity; and (ii) Polydispersity increases with concentration.

The major disadvantage of cumulants analysis is that it does not give details con-

\section{TABLE II}

Cumulants for Systems Having Water-to-Surfactant Concentration Ratios $N_{\mathrm{w}} / N_{\mathrm{aot}}=12$ and $N_{\mathrm{w}} / N_{\mathrm{aot}}=4.2$

\begin{tabular}{|c|c|c|c|c|}
\hline Sample & $\begin{array}{l}\text { Volume } \\
\text { fraction }\end{array}$ & $\begin{array}{l}\text { Diffusivity } \\
\text { coefficient } \\
\left(D \times 10^{6}\right. \\
\left.\mathrm{cm}^{2} / \mathrm{sec}\right)\end{array}$ & Variance & $\begin{array}{l}\text { Hydro- } \\
\text { dynamic } \\
\text { radius, } R_{\mathrm{h}}\end{array}$ \\
\hline \multicolumn{5}{|l|}{$N_{\mathrm{w}} / N_{\mathrm{aot}}=12$} \\
\hline \multirow[t]{7}{*}{$0 \mathrm{wt} \% \mathrm{NaCl}$} & 0.455 & 1.26 & 0.15 & 45.0 \\
\hline & 0.303 & 1.12 & 0.07 & 50.4 \\
\hline & 0.227 & 1.06 & 0.08 & 53.6 \\
\hline & 0.091 & 1.15 & 0.07 & 49.2 \\
\hline & 0.041 & 1.35 & 0.04 & 41.7 \\
\hline & 0.022 & 1.54 & 0.05 & 36.7 \\
\hline & 0.015 & 1.69 & 0.05 & 33.5 \\
\hline \multirow[t]{6}{*}{$3 \mathrm{wt} \% \mathrm{NaCl}$} & 0.455 & 1.45 & 0.197 & 39.0 \\
\hline & 0.303 & 1.61 & 0 & 35.2 \\
\hline & 0.227 & 1.61 & 0 & 34.8 \\
\hline & 0.091 & 1.66 & 0 & 34.0 \\
\hline & 0.041 & 1.64 & 0 & 34.5 \\
\hline & 0.022 & 1.76 & 0 & 32.0 \\
\hline \multicolumn{5}{|l|}{$N_{\mathrm{w}} / N_{\mathrm{aot}}=4.2$} \\
\hline $0 \mathrm{wt} \% \mathrm{NaCl}$ & 0.14 & 1.52 & 0.06 & 37.3 \\
\hline $3 \mathrm{wt} \% \mathrm{NaCl}$ & 0.14 & 2.21 & 0.043 & 25.5 \\
\hline $7 \mathrm{wt} \% \mathrm{NaCl}$ & 0.14 & 2.61 & 0.028 & 21.7 \\
\hline $11 \mathrm{wt} \% \mathrm{NaCl}$ & 0.14 & 2.65 & 0.021 & 21.4 \\
\hline $0 \mathrm{wt} \% \mathrm{NaCl}$ & 0.023 & 2.25 & 0.021 & 25.1 \\
\hline $3 \mathrm{wt} \% \mathrm{NaCl}$ & 0.023 & 2.57 & 0 & 21.9 \\
\hline $7 \mathrm{wt} \% \mathrm{NaCl}$ & 0.023 & 2.65 & 0 & 21.3 \\
\hline $11 \mathrm{wt} \% \mathrm{NaCl}$ & 0.023 & 2.61 & 0 & 21.6 \\
\hline
\end{tabular}

cerning the shape of a distribution. Only the mean and variance can be extracted as useful parameters. Alternatively, we can attempt to fit the same data to a sum of two exponentials, as suggested by Pusey et al. (38), requiring the distribution to be two delta functions. This method only gave physically meaningful results with high volume fraction samples. For samples at low volume fraction and most of the systems containing salt, the second exponential developed a negative characteristic decay time. For the samples at high volume fraction, the self diffusion coefficient is a decreasing function of volume fraction, as expected $(38,39)$.

Histogram analysis is sensitive to asymmetry and does not force the distribution to exhibit a particular shape. The physical significance of the asymmetries observed in the present data supports a strong case for the idea that the microemulsion droplets attract each other and that the $\mathrm{NaCl}$ moderates these interactions. Figures 6 and 7 show the results of histogram analysis for systems with no salt and $3 \%$ salt (water-to-AOT ratio $=12$ ), respectively. These results are reported in hydrodynamic radius space. The curve fits were conducted according to procedures we

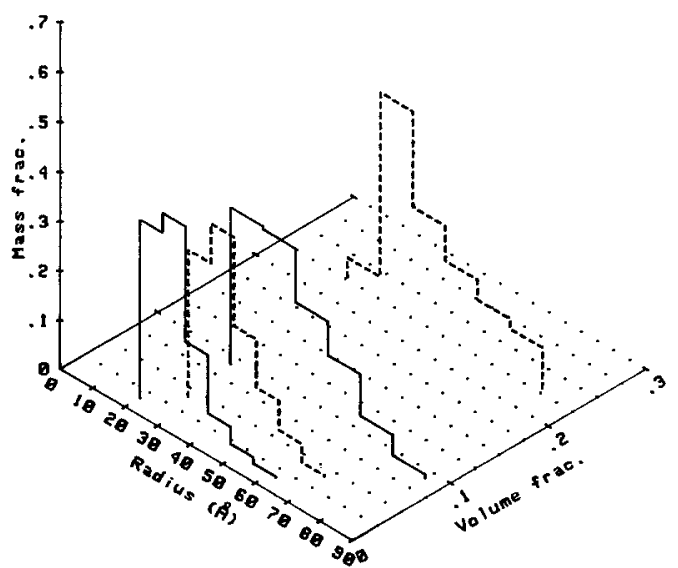

FIG. 6. Histogram distributions for the W/O microemulsions with no salt and water-to-AOT ratio of 12 as a function of disperse phase volume fraction. Radii of the microemulsion droplets were calculated from the autocorrelation function of the scattered light as described in Ref. (27). 


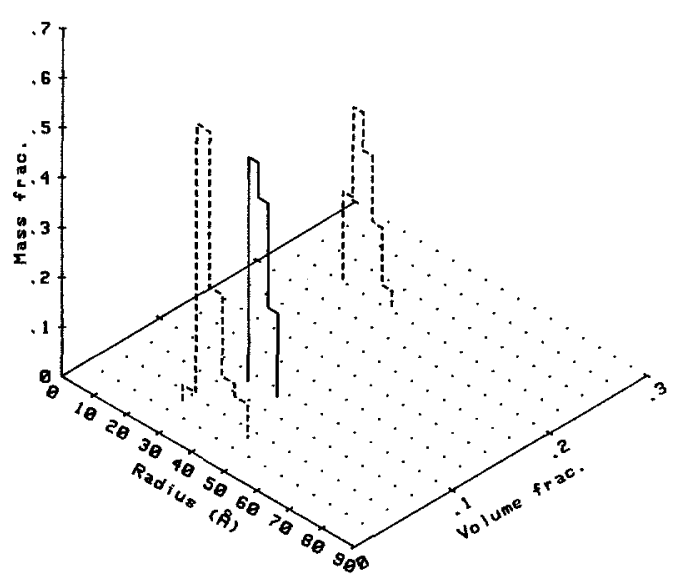

FIG. 7. Histogram distributions for the W/O microemulsions with $3 \%$ salt and water-to-AOT ratio of 12 as a function of disperse phase volume fraction.

have developed elsewhere (40). For the systems with no salt, there is a clear trend in the shape of the distribution with volume fraction. At high volume fraction (0.227), there is a maximum near $35 \AA$ and a significant contribution from larger hydrodynamic sizes. As the volume fraction of dispersed phase decreases, this contribution from larger sizes (or slower modes) is diminished, but the maximum in the distribution stays at about the same location, suggesting that the same small droplet size is preserved at high concentration. For the salted systems, the distributions are narrow, relatively insensitive to volume fraction, and centered near the same point. The contributions observed at higher values of hydrodynamic radius with the unsalted systems could be due to the formation of aggregates or to the cooperative motion of droplets interacting at long range. Either of these effects would be more pronounced with the closer interparticle spacing achieved at high volume fraction.

The same trends with volume fraction and salt content are observed with histogram analysis of the systems with water-to-AOT ratio of 4.2. The maximum in the distribution does not shift appreciably, but rather the tail on the distribution disappears with the ad-

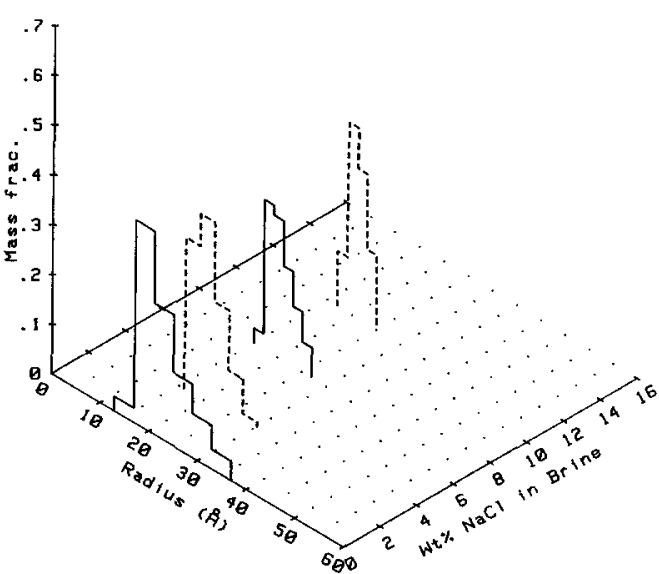

FIG. 8. Histogram distributions for the microemulsions with water-to-AOT ratio of 4.2 series as a function of salt content at disperse phase volume fraction $=0.023$.

dition of $\mathrm{NaCl}$. Figure 8 shows the same behavior for salt added to systems with volume fraction equal to 0.023 . As expected, these distributions are somewhat narrower than those at higher volume fraction.

In order to test the effect of different amounts of water on microemulsions of constant surfactant to salt molar ratio $\left(N_{\mathrm{aot}} / N_{\mathrm{e}}\right)$ a new series of microemulsions were prepared. Figure 9 shows the results of cumulants analysis on DLS experiments performed with these systems (reported as average hydrodynamic radius). The results show that at low water content, the droplets behave more like

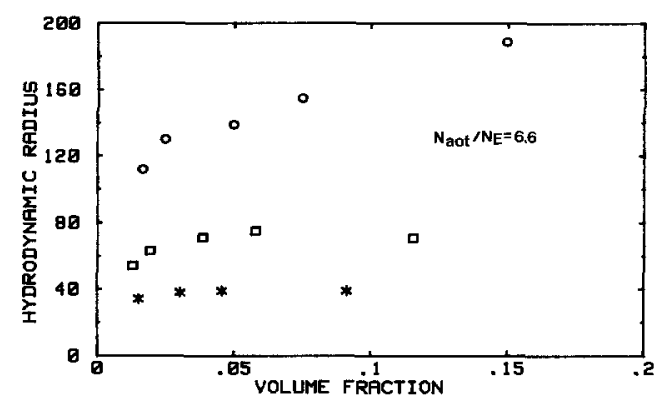

FIG. 9. Hydrodynamic radius vs total solute concentration when the surfactant-to-salt ratio is kept constant at 6.6. Water-to-AOT ratios are $10(*), 20(\square)$, and 34 (0). 
hard spheres than at high water content. This is apparent from the onset of volume fraction dependence with increasing water content.

This behavior suggests that the $\mathrm{NaCl}$ is dispersed uniformly in the droplet core and does not have a preferential interaction with the interior surface occupied by the surfactant. If the systems with constant salt-to-surfactant ratio had all exhibited similar trends with volume fraction, we might have concluded that the $\mathrm{NaCl}$ was incorporated into the surface. In addition to the increase in volume fraction dependence with water content, there was an increase in polydispersity. Variances for the systems with water-to-AOT ratio of 10 were 0 , while the variances for the systems with a ratio of 34 ranged from 0.15 to 0.30 .

There are several alternatives for explaining the way in which salt alters the characteristic droplet and its interactions. Because $\mathrm{NaCl}$ exists as ionic species in aqueous solutions and is insoluble in heptane, we expect the $\mathrm{NaCl}$ in the W/O microemulsion to be dispersed in the droplet water core. Any explanation for the effects we observe must incorporate this idea.

Extra electrolyte in the core could salt out other solutes. It could reduce the number of surfactant molecules which dissociate to form ions by competing for water of hydration. It could reduce the solubility of surfactant molecules, dissociated or undissociated, in the core. For the charged surfactant head groups, which occupy the surface, the electrolyte can provide extra screening, diminishing repulsive electrostatic interactions and allowing for a tighter, perhaps more organized, packing of surfactant molecules in the interface.

All the mechanisms for attractive interactions between droplets, which seem feasible, could be affected by the addition of $\mathrm{NaCl}$. Organization of the surfactant chains as a secondary effect of the organization of ionic head groups through screening could lead to an alteration in the type of interaction suggested by Lemaire $e t$ al. (20). The orientation and packing of nonpolar tails with salt ad- dition might diminish the probability of overlap between droplet surfaces. Reversible coalescence events which involve communication between droplet cores, over and above surface interactions, might be reduced by an increase in the rigidity of the interfacial layer through the addition of $\mathrm{NaCl}$.

An alternative mechanism is electrostatic interactions between droplets. If some of the droplets develop an occasional charge through exchange of electrolyte or if the individual droplets develop asymmetric electric fields (dipoles) through fluctuations in the relative positions of charged species within the neutral droplet, then attraction could be electrostatic. In either of these cases, an increase in the number of charged species per droplet with the addition of $\mathrm{NaCl}$ would decrease the magnitude of fluctuations and lead to a reduction in the attractive force.

In order to estimate, semiquantitatively, the magnitude of the attractive force between microemulsion droplets in heptane we used the fluctuating dipole model. This model is analog of the London (41) model for the van der Waals force. Just like the electrons moving around the fixed positive center of an atom, in the W/O microemulsion droplet positively charged ions diffuse inside the water core surrounded by the negatively charged interface containing the surfactant head groups. By using reasonable values for the diffusion coefficient of the positive ion, the polarizability and the dipole moment we were able to estimate the Hamaker constant for the microemulsion droplets in heptane.

In Table III we report the values of the experimentally determined van der Waals constants calculated from the light scattering data using (23)

$$
\begin{aligned}
A=\frac{\pi}{9} A_{131}\left(2 R_{\mathrm{w}}\right)^{3}\{1 / 2 & \ln \left(\frac{s-1}{s+1}\right) \\
& \left.-s-s^{3} \ln \left(\frac{s^{2}-1}{s^{2}}\right)\right\},
\end{aligned}
$$

where

$$
R_{\mathrm{w}}=\text { water core radius }=R_{\mathrm{h}}-l_{\mathrm{c}}
$$


TABLE III

Experimental and Estimated Hamaker Constants ${ }^{a}$

\begin{tabular}{ccccc}
\hline$\% \mathrm{NaCl}$ & $\begin{array}{c}R_{\mathrm{h}} \\
(\AA)\end{array}$ & $\begin{array}{c}R_{\mathrm{ma}} \\
(\AA)\end{array}$ & $\begin{array}{c}A \\
\left(\times 10^{\circ} \mathrm{cm}^{3} \mathrm{erg}\right)\end{array}$ & $\begin{array}{c}A_{11} \\
\left(\times 10^{11} \mathrm{erg}\right)\end{array}$ \\
\hline 0 & 29 & 24 & 8.2 & 0.84 \\
1 & 29 & 21.5 & 5.4 & 0.37 \\
3 & 29 & 21 & 2.9 & 0.20
\end{tabular}

Predictions for the Fluctuating dipole model

\begin{tabular}{|c|c|c|}
\hline $\begin{array}{c}R_{w} \\
(\AA, \text { core radius })\end{array}$ & $\begin{array}{c}d \\
(\AA \AA \\
\AA\end{array}$ & $\begin{array}{c}A_{31} \\
\left(\times 10^{11} \text { erg }\right)\end{array}$ \\
\hline 20 & 5 & 1.8 \\
\hline 20 & 4 & 0.48 \\
\hline 20 & 3 & 0.08 \\
\hline 25 & 5 & 0.48 \\
\hline 25 & 4 & 0.13 \\
\hline 25 & 3 & 0.02 \\
\hline
\end{tabular}

${ }^{a}$ Experimental data is for microemulsion systems with a water-to-AOT ratio of 8.4 .

$$
\begin{gathered}
\left(l_{\mathrm{c}}=11 \AA\right) \\
s=R_{\mathrm{hs}} / R_{\mathrm{w}} .
\end{gathered}
$$

We can eliminate the solvent effect using (45)

$$
A_{131}=A_{11}+A_{33}-2 \sqrt{A_{11}} \cdot \sqrt{A_{33}}
$$

where

$A_{131}=$ Hamaker constant of droplets

in heptane

$A_{33}=$ Hamaker constant of heptane

$A_{11}=$ Hamaker constant of droplets.

Also given are the predictions of the fluctuating dipole model. These results agree within an order of magnitude. The dipole length was approximated by the Debye length, which for a simple $3 \% \mathrm{NaCl}$ solution is about $4.2 \AA$. From the experimental data we see that salt decreases the Hamaker constant or reduces attractive forces. This is in agreement with the prediction of the model because the dipole length will decrease as the electrolyte concentration is increased. We should also note that the Hamaker constants given in Table III are in good agreement with the only other reported value of $1.75 \times 10^{-12}$ erg (23) and close to two orders of magnitude larger than values from other sources (42). Clearly the origins of the two types of attractive forces differ and the long range attractive forces are too weak to account for the large Hamaker constants observed.

Several unresolved questions disallow anything more than an order of magnitude analysis. (i) The number of surfactant head groups which are dissociated is unknown, hence an appropriate value for $d$ can only be guessed. The addition of salt would influence this number. (ii) The electronic environment of the core is probably more complicated than a simple electrolyte. (iii) This attractive force could have a component which is due to orientation and/or induction effects as well as dispersion, if these dipoles are "long-lived" relative to the characteristic time of the droplet motion.

All of the above interaction mechanisms are plausible and might be moderated by the addition of $\mathrm{NaCl}$. Clarification is yet to come.

Two other observations, concerning the effect of electrolyte on the phase behavior of these W/O microemulsions, are worthy of note in this context. First, the time required to form a stable microemulsion with $\mathrm{NaCl}$ brine is much longer than that required with pure water, as discussed above. We have shown that the characteristic droplet develops more slowly with $\mathrm{NaCl}$ in the system (28). If $\mathrm{NaCl}$ hardens the interface, then the evolution of droplets to their equilibrium condition might be limited by mass transfer across this more substantial boundary. If the droplet cores communicate through coalescence events in the unsalted system and not in the salted, then the more rapid droplet growth and evolution to equilibrium are easily explained. This slower evolution might also be due to a similarity in chemical potential between the aqueous solution in the droplet and the undispersed aqueous phase, reducing the "driving force" for mass transfer across the interface. The second observation is that 
salt reduces the maximum solubility of the aqueous phase, as evidenced by the solubilization behavior of systems with water-toAOT ratio of 12 above and the observations of Kuneida and Shinoda (1) discussed above.

\section{CONCLUSIONS}

We have shown that $\mathrm{NaCl}$ added to $\mathrm{W} / \mathrm{O}$ microemulsions of AOT in heptane moderates the interaction between suspended droplets. Viscosity measurements show clearly that with the addition of $\mathrm{NaCl}$, the system behaves like a suspension of hard spheres. Analysis of average intensity light scattering data shows that salt diminishes the magnitude of attractions between particles. DLS experiments also show that the salted systems are more nearly hard-sphere suspensions than the unsalted systems. The polydispersity observed in the unsalted systems is consistent with the notion of attractive interactions.

There is some evidence that the assumption of a continuum insensitive to the addition of electrolyte is in error. The curvature in the colligative region for the average intensity experiments with unsalted systems suggests that the characteristic particle size may decrease at low concentration due to the loss of water and/or surfactant to the continuum.

The exact mechanism by which electrolyte alters the characteristic microemulsion macroparticle is not clear. Several explanations, which are not mutually exclusive, are consistent with what we have observed.

\section{ACKNOWLEDGMENTS}

Partial support of this research by the National Science Foundation (Grants CPE-8303850, CPE-8107724, and DMR-8100130) and by the donors of the Petroleum Research Fund administered by the American Chemical Society (PRF 13210-AC5, 7-C) is gratefully acknowledged.

\section{REFERENCES}

1. Kuneida, H., and Shinoda, K., J. Colloid Interface Sci. 70, 577 (1979).

2. Kon-no, K., and Kitahara, A., J. Colloid Interface Sci. 41, 47 (1972).
3. Kitahara, A., and Kon-no, K., J. Phys. Chem. 70(11), 3394 (1966)

4. Aebi, C. M., and Wiebush, J. R., J. Colloid Sci. 14, 161 (1959).

5. Eicke, H. F., Helv. Chim. Acta 62(2), 448 (1979).

6. Magid, L. J., Kon-no, K., and Martin, C. A., J. Phys. Chem. 85(10), 1434 (1981).

7. Wentz, M., Smith, W. H., and Martin, A. R., $J$. Colloid Interface Sci. 29, 36 (1969).

8. Eicke, H. F., J. Colloid Interface Sci. 68(3), 440 (1979).

9. Zulauf, M., and Eicke, H. F., J. Phys. Chem. 83(4), (1980).

10. Tamamushi, B., and Watanabe, N., Colloid Polym. Sci. 258, 174 (1980).

11. Day, R. A., Robinson, B. H., Clarke, J. H., and Doherty, J. V., J. Chem. Soc. Faraday Trans. 75, 132, (1979).

12. Eicke, H. F., and Shepherd, J. C. W., Helv. Chim. Acta 57(7), 1951 (1974).

13. Eicke, H. F., Shepherd, J. C. W., and Steineman, A. J., J. Colloid Interface Sci. 56(1), 168 (1975).

14. Fletcher, P. D. I., and Robinson, B. H., Ber. Bunsenges. Phys. Chem. 85, 863 (1981).

15. Eicke, H. F., and Markovic, Z., J. Colloid Interface Sci. 85(1), 198 (1982).

16. Wong, M., Thomas, J. K., and Nowak, T., J. Amer. Chem. Soc. 99(14), 4730 (1977).

17. Graciaa, A., Lachaise, J., Chabrat, P., Letamendia, L., Rouch, J., Vaucamps, C., Bourrel, M., and Chambu, C., J. Phys. Lett. 38, 253 (1977).

18. Cazabat, A. M., Langevin, D., and Pouchelon, A., J. Colloid Interface Sci. 73(1), 1 (1980).

19. Cazabat, A. M., and Langevin, D., J. Chem. Phys. 74,(6), 3148 (1981).

20. Lemaire, B., Bothorel, P., and Roux, D., J. Phys. Chem. 87(6), 1023 (1983).

21. Brunetti, S., Roux, D., Bellocq, A. M., Fourche, G., and Bothorel, P., J. Phys. Chem. 87(6), 1028 (1983).

22. Cazabat, A. M., Chatenay, D., Langevin, D., and Pouchelon, A., J. Phys. Lett. 41, 441 (1980).

23. Calje, A. A., Agterof, W. G. M., and Vrij, A., in "Micellization, Solubilization and Microemulsions" (K. L. Mittal, Ed.), Vol. 2. Plenum, New York, 1977.

24. Carnahan, N. F., and Starling, K. E., J. Chem. Phys. 51, 635 (1969).

25. Koppel, D. E., J. Chem. Phys. 57(11), 4814 (1972).

26. Pike, E. R., in "Scattering Techniques Applied to Supramolecular and Nonequilibrium Systems" (S.-H. Chen, B. Chu, and R. Nossal, Eds.). Plenum, New York, 1980.

27. Gulari, E., Gulari, E., Tsunashima, Y., and Chu, B., J. Chem. Phys. 70, 2965 (1979).

28. Bedwell, W. B., and Gulari, E., in "Solution Behavior 
of Surfactants" (K. L. Mittal and E. J. Fendler, Eds.), p. 833. Plenum, New York, 1982.

29. Anderson, J. L., and Reed, C. C., J. Chem. Phys. 64(8), 3240 (1976).

30. Mooney, M., J. Colloid Sci. 6, 162 (1950).

31. Vand, V., J. Phys. Colloid Chem. 52, 277 (1952).

32. Roscoe, R., Brit. J. Appl. Phys. 3, 267 (1952).

33. Brinkman, H. C., J. Chem. Phys. 20, 571 (1952).

34. Rutgers, R., Rheol. Acta 2, 305 (1962).

35. Goodwin, J. W, "Specialist Periodical Report on Colloid Science," Vol. 2, pp. 246-269. The Chemical Society, Burlington House, London, 1975.
36. Cabos, C., and Delord, P., J. Phys. Lett. 41, L-455 (1980).

37. Lalanne, J. R., Pouligny, B., and Sein, E., J. Phys. Chem. 87, 696 (1983).

38. Cebula, D. J., Ottewill, R. H., Ralston, J., and Pusey, P. N., J. Chem. Soc. Faraday Trans. 177,2585 (1981).

39. Pusey, P. N., Fijnaut, H. M., and Vrij, A., J. Chem. Phys. 77(9), 4270 (1982).

40. Bedwell, W. B., Gulari, E., and Melik, D., in "Measurement of Suspended Particles by Quasi-elastic Light Scattering" (B. E. Dahneke, Ed.). Wiley, New York, 1983.

41. London, F., Trans. Faraday Soc. 33, 8 (1937).

42. Visser, J., Adv. Colloid Interface Sci. 3, 331 (1972). 